International Journal of Ophthalmology \& Eye Science (IJOES)

ISSN 2332-290X

\title{
Ophthalmic Plastic, Reconstructive and Cosmetic Eyelid Surgeries in Taiwan
}

\begin{abstract}
Hsu $\mathrm{WM}^{1,2^{*}}$
${ }^{1}$ Department of Ophthalmology, College of Medicine, School of Medicine, Taipei Medical University, Taipei, ROC, Taiwan.

${ }^{2}$ Department of Ophthalmology, Taipei Medical University- Shuang Ho Hospital, New Taipei City, ROC, Taiwan.
\end{abstract}

\section{*Corresponding Author:}

Dr. Wen-Ming Hsu,

Professor of Ophthalmology, Department of Ophthalmology, Taipei Medical University - Shuang Ho Hospital. \#291, Zhong Zheng Road, Zhonghe District, New Taipei City 23561, ROC, Taiwan.

Tel: +886-2-2393.9351

Fax: +886-2-2327.9097

E-mail:wmhsu3939@gmail.com

Received: May 12, 2015

Accepted: June 22, 2015

Published: July 02, 2015

Citation: Hsu WM (2015) Ophthalmic Plastic, Reconstructive and Cosmetic Eyelid Surgeries in Taiwan. Int J Ophthalmol Eye Res. S4:001, 1-4. doi: http://dx.doi.org/10.19070/2332-290X-SI04001

Copyright: Hsu $\mathbf{W M}^{\odot}$ 2015. This is an open-access article distributed under the terms of the Creative Commons Attribution License, which permits unrestricted use, distribution and reproduction in any medium, provided the original author and source are credited.

\section{Background}

Taiwan, a medium-sized island located south-east of mainland China, has a land area of 36,000 square kilometers and a population of 23.4 millions. More than $11.7 \%$ of its total population is older than 65 years. In 2013, the average life expectancy in Taiwan was 79.16 years (male 76.72 years, female 83.20 years). For the past 60 years, Taiwan has benefited from the implementation of democracy, universal education, good law and order, improved economic conditions. Taiwan has become a free and open society with a large number of medical manpower to provide excellent medical care. Taiwan's government in 1995 began implementing the National Health Insurance for its citizens. In 2013, the total insurance expenditure of the National Health Insurance was 500 billions NT dollars (15.8 billion USD), accounting for $6.1 \%$ of the GDP [1].

In recent years, the demand for plastic and cosmetic surgeries has increased. Many of the plastic, reconstructive and cosmetic surgeries are focused on the region of eyes and face.

\section{Development of ophthalmic plastic, cosmetic and reconstructive surgery in Taiwan}

Ophthalmic Plastic and Reconstructive Surgery (OPRS) is a relatively new entity, but the art has been practiced in one form or another from time immemorial. Reparative procedures had been recorded in ancient China and India as well as in Celsus. Before
1970, most ophthalmic plastic surgeries were performed by general ophthalmologists in Taiwan. After 1970, and the ophthalmic plastic section became one of the 5 essential sections of ophthalmology department in most medical centers since 1980. Many hospitals appreciated this new section and sent many ophthalmologists abroad to learn ophthalmic plastic and reconstructive surgeries [2].

From 1986 to 2000, approximately 40 ophthalmic plastic surgeons underwent their subspecialty training program (i.e., ophthalmic plastic and orbital surgery) in the USA: (such as: Manhattan Eye Ear Throat Hospital, New York Eye and Ear Infirmary, University of California - Los Angeles, University of California - Davis , University of California - San Francisco, University of Southern California, Duke University, University of Utah, University of Iowa, Massachusetts Eye and Ear Infirmary, Wills Eye Hospital, Wilmer Eye Institute, Bascom Palmer Eye Institute); in Canada: (University of British Columbia); in England: (Moorfields Eye Hospital); and in Japan and Korea. Some 70 ophthalmic plastic surgeons underwent their subspecialty training in medical centers throughout Taiwan [2].

The Taiwan Society of Ophthalmic Plastic and Reconstructive Surgery (TSOPRS), founded in October 2013 (Figure.1) [2], provided academic activities, continuing educational courses (such as: symposium, workshop and live surgery demonstration) for residents and practicing ophthalmologists to learn ophthalmic plastic and reconstructive surgery. The society member has increased from the initial 67 in 2013 to a total of 112 in 2015 [2].

From 1985 to 2014, Taiwan ophthalmologists have published 312 academic papers (76 articles in Science Citation Index [SCI] journals and 236 in non-SCI journals) in the field of ophthalmic plastic and reconstructive surgery. Many of the publications bring meaningful and important topics into light [3-9].

\section{Workforce involving ophthalmic plastic and re- constructive surgery in Taiwan}

In 2014, there were a total 1,728 ophthalmologists in Taiwan serving a population of 23.4 million people [10]. Among the ophthalmologists, 112 are defined as oculo-plastic surgeons $(6.5 \%$ of the total 1,728 ophthalmologists) [2,10].

In Taiwan, specialists such as plastic surgeons, dermatologists, oral surgeons, otolaryngologists, family medicine doctors and even gynecologists perform many eyelid aesthetic procedures (especially filler injection). Plastic surgeons are the predominant 
Figure 1. Inaugural meeting of the Taiwan Society of Ophthalmic Plastic and Reconstructive Surgery. (October. 10, 2013)

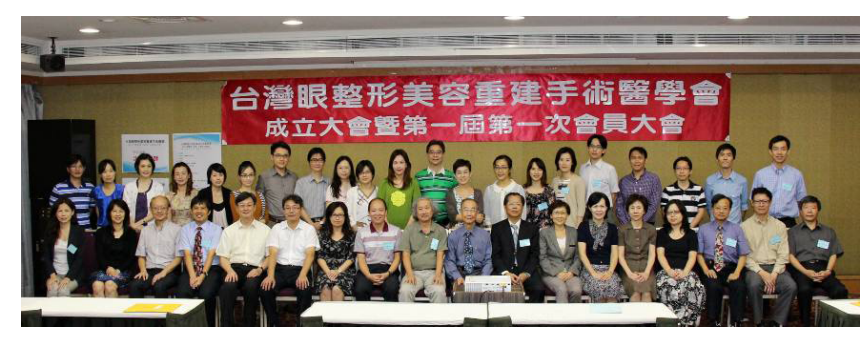

group among these physicians. Furthermore, approximately 25,000 paramedical persons, nurses and technicians assist in these plastic and cosmetic operations $[2,11]$.

\section{Ophthalmic plastic, reconstructive surgeries and periocular aesthetic procedures in Taiwan}

Entropion correction was the most common oculoplastic surgeries performed in Taiwan. Table 1 lists the approximate number of major ophthalmic plastic, orbital and reconstructive surgeries performed annually (2003-2013) by Taiwan ophthalmologists [2, $11,12]$.

In 1979, intubation DCR (Dacryo-Cysto-Rhinostomy) technique was introduced to Taiwan [13]. Some modification techniques for improving success rate of external DCR were reported [14-16]. Endoscopic DCR have been promoted since 2009. Now (2013 to 2015), two thirds of DCR procedures were performed through endoscopic approach. Some Taiwanese oculo-plastic surgeons are interested in the field of TED (Thyroid Eye Diseases) and their researches were well documented [17-21].

Botulinum injection was introduced to Taiwan in 1999 [11]. Initially, it was applied to treat blepharospasm or eyelid retraction, now it was widely applied to some cosmetic procedures. Filler injection was introduced to Taiwan in 2005 [12]. Many instructional teaching courses were held to teach physicians how to perform botulinum and filler injection.

The exact number of aesthetic eyelid surgery cannot be easily documented. However, the estimated number of upper blepharoplasty procedures performed in Taiwan is approximately 60,00080,000 procedures per year, and the number of lower blepharoplasty is approximately $40,000-50,000$ procedures per year. Plastic surgeons performed most aesthetic eyelid surgeries in more than
$60 \%$ of the patients, whereas ophthalmologists perform less than $20 \%$ of these surgeries. Physicians from other specialties such as dermatologists, family medicine account for the remaining $20 \%$ $[11,12]$.

A survey performed in 2010 showed that "botulinum toxin injection" is the most common aesthetic procedure (32.7\%), followed by hyaluronic acid injection $(20.1 \%$ ) performed in Taiwan. [22] In peri-ocular aesthetic procedures, there are approximately 400,000 botulinum toxin injections and 160,000 hyaluronic acid injections per year [22]. Among these injection procedures, plastic surgeons performed approximately $40 \%$ of these cases, whereas ophthalmologists performed approximately $30 \%$ of these procedures. Doctors from other fields account for the remaining $30 \%[10,11]$.

\section{Obstacles of ophthalmic plastic, reconstructive and cosmetic eyelid surgery in Taiwan}

In addition to competition and conflicts between ophthalmologists and other specialists in cosmetic eyelid surgeries, the operation fee in ophthalmic plastic and reconstructive surgery is another obstacle [1]. The operation fee in cosmetic eyelid surgery is "out of pocket" by patient (for example, the fee of one site upper blepharoplasty is around $\$ 9,000-20,000$ NTD, or $\$ 300-650$ USD, by individualized surgeon), but the operation fee of functional (non- cosmetic) oculoplastic surgeries were paid by government insurance (NHI of Taiwan) [1].

Table 2 lists the operation fee (under the control of the NHI of Taiwan) for some ocular surgeries [1]. As the data show, the operation fee for ophthalmic plastic, orbital and reconstructive surgery is relatively low, compared to the payment for intraocular procedures such as cataract surgery, penetrating keratoplasty, and trabeculectomy [1].

The payment for levator muscle resection is $\$ 5,820$ New Taiwan

Table 1. Some major oculoplastic and reconstructive operations performed annually (2003-2013) by Taiwan ophthalmologists $[1,2,11,12]$.

\begin{tabular}{|c|c|}
\hline Operation & Approximate numbers per year \\
\hline Entropion Correction & 7,200 \\
\hline Ptosis correction operation & 3,500 \\
\hline Excision of benign lid tumor & 4,500 \\
\hline Excision of malignant lid tumor with reconstruction & 90 \\
\hline Dacryo-Cysto-Rhinostomy (DCR) & 250 \\
\hline Conjunctivo - DCR & 40 \\
\hline Orbital tumor removal (or biopsy) & 180 \\
\hline Orbital fracture repair (combined with plastic surgeons) & 120 \\
\hline
\end{tabular}


Table 2. Operation fee of eye operation in Taiwan (NHI standard fee, year 2013).

\begin{tabular}{|c|c|}
\hline Eye operation & Operation Fee, NTD (USD) ${ }^{a}$ \\
\hline Cataract surgery (ECCE, Phaco) & $9,000(286)$ \\
\hline Penetrating keratoplasty & $12,390(393)$ \\
\hline Trabeculectomy (glaucoma) & $6,939(220)$ \\
\hline Ptosis (levator resection/advancement) & $5,820(185)$ \\
\hline Ptosis (frontalis sling) & $5,449(173)$ \\
\hline Eyelid tumor (removal) & $1,651(48)$ \\
\hline (with reconstruction) & $6,989(221)$ \\
\hline Entropion (eyelid) & $3,324(106)$ \\
\hline Ectropion & $4,070(129)$ \\
\hline Dacryo-cysto-rhinostomy & $7,760(246)$ \\
\hline Conjunctivo-DCR & $8,240(262)$ \\
\hline Orbitotomy (tumor removal, lateral) & $13,109(416)$ \\
\hline Orbital floor repair & $8,163(259)$ \\
\hline Enucleation of eyeball & $6,783(214)$ \\
\hline Evisceration of eyeball & $5,946(189)$ \\
\hline
\end{tabular}

$\mathrm{DCR}=$ dacryocystorhinostomy, NTD $=$ new Taiwan dollars, USD $=$ United States dollars

a : The information in this column is from the National Health Insurance Statistics and Survey (in Chinese) published by the Bureau of National Health Insurance, Ministry of Health and Welfare in Taipei, Taiwan. Copyright holder: National Health Insurance, Administration Ministry of Health and Welfare, 2013

Dollar (NTD), which is nearly two-third of the payment for cataract surgery ( $\$ 9,000 \mathrm{NTD})$ [1]. In the USA, operation fee for cataract surgery and levator muscle resection is nearly the same (\$700-800 USD) [23]. The surgical fee for ophthalmic surgeries in Taiwan is even lower than fees paid through Medicare for a similar procedure in the USA. For example, the operation fee for levator muscle resection was nearly one-fourth of the operation fee (\$190 USD, in Taiwan vs. $\$ 750$ USD in USA) [1, 23]. The relatively low payment for ophthalmic plastic surgeries in Taiwan should be considered an important issue that hinders ophthalmologists from performing ophthalmic plastic surgeries.

\section{Future of ophthalmic plastic, reconstructive and cosmetic surgery in Taiwan}

In order to overcome the above obstacles and enhance further development in the field of ophthalmic plastic and reconstructive surgery, the Taiwan Society of Ophthalmic Plastic and Reconstructive Surgery have held many symposia and seminars, in the recent 3 years, to provide opportunities for general ophthalmologists to learn more cosmetic surgeries and aesthetic procedures. There are also many combined meetings among ophthalmology, plastic surgery and dermatology to exchange their experiences. With the cooperation between ophthalmologists, plastic surgeons, dermatologists, the future of ophthalmic plastic, reconstructive and cosmetic surgery in Taiwan will be bright.

\section{Conclusion}

This article briefly describes the development and current status of ophthalmic plastic and reconstructive surgery in Taiwan. Up to 2015, there are 112 ophthalmic plastic surgeons in Taiwan. They have had their fellowship training abroad (most of them trained in the USA) or in Taiwan. All ophthalmic plastic surgeons were well trained and capable of performing major ophthalmic plastic surgeries. The service level of oculoplastic and reconstructive surgeries become higher and higher in recent 30 years. The payment rates by Taiwan National Health Insurance for ophthalmic plastic and reconstructive surgeries are relatively low. Competition and conflict of interest among ophthalmologists, plastic surgeons, and other surgeons is an issue in Taiwan.

\section{Acknowledgments}

The author thanks the Bureau of National Health Insurance (Taiwan Government) for their data and Taiwan Medical Association for the Statistics information.

The author also thanks I-Chan Lin, M.D., Chi-Hsin Hsu, M.D., Yun-Dun Shen and Allen Lin, M.D., for their assistance in the collection of some data and correction of the manuscript.

\section{References}

[1]. Bureau of National Health Insurance (2012) The National Health Insurance statistics and survey [in Chinese]. Taipei, Taiwan, Executive Yuan, R.O.C.: Bureau of National Health Insurance Department of Health. Can be accessed at: http://www.nhi.gov.tw

[2]. Hsu WM (2013) Bulletin of Taiwan Society of Ophthalmic Plastic and Reconstructive Surgery [in Chinese] Taipei, Taiwan, Taiwan Academy of Ophthalmology.

[3]. Hsu WM, Liu D (1985) A new approach to the correction of involutional entropion by pretarsal orbicularis oculi muscle fixation. Am J Ophthalmol 100(6): 802-805.

[4]. Ma L, Wong SP, Wu CY, Yao SJ (1987) Orbital reconstruction with proplast. Ophthal Plast Reconstr Surg 3(3): 151-157.

[5]. Hsu WM, Wu JS (1990) Global oculoplastic and eyelid problems. Curr Opinion Ophthalmol 1(5): 506-512.

[6]. Hsu WM, Chung C (2002) Acupuncture in the treatment of blepharospasm. Acta Soc Ophthalmol Sinicae 41: 114-116.

[7]. Liao SL, Kao SC, Tseng JH, Lin LL (2005) Surgical coverage of exposed hydroxyapatite implant with retroauricular myoperiosteal graft. $\mathrm{Br} \mathrm{J}$ Ophthalmol 89(1): 92-95.

[8]. Huang YH, Liao SL, Lin LL (2009) Balloon dacryocystoplasty and monocanalicular intubation with Monoka tubes in the treatment of congenital 
nasolacrimal duct obstruction. Graefes Arch Clin Exp Ophthalmol 247(6): 795-799.

[9]. Wu SY, Ma L, Chen RJ, Tsai YJ, Chu YC (2010) Analysis of bicanalicular nasal intubation in the repair of canalicular lacerations. Jpn J Ophthalmol 54(1): 24-31.

[10]. Taiwan Medical Association (2012) Statistics Yearbook of Practicing Physicians and Health Care Organizations in Taiwan (2002 to 2012). Taiwan Medical Association, Taipei.

[11]. Hsu WM (2007) Status of oculoplastic surgery in Taiwan. In: Inaugural Scientific Meeting of the Taiwan Society of Ophthalmic Plastic and Reconstructive Surgery. Taipei, Taiwan, Taiwan Academy of Ophthalmology.

[12]. Hsu WM (2012) Ophthalmic plastic and orbital surgery in Taiwan. In: Symposium on current and future of oculoplastic and orbital surgery of the Chinese Medical Society - Oculoplastic Branch Society of Chinese Ophthalmological Society, Shanghai, China.

[13]. Hsu WM, JH Liu (1988) Intubation dacryocystorhinostomy - 13 years experience. Trans Soc Ophthalmol Sinicae 27: 515-520.

[14]. Kao SCS, Liao SL, Tseng JHS, Chen MS, Hou PK (1997) Dacryocystorhinostomy with intraoperative mitomycin C. Ophthalmology 104(1): 86-91.

[15]. Liao SL, Kao S, Tseng J, Chen M, Hou P (2000) Results of intraoperative mitomycin $\mathrm{C}$ application in dacryocystorhinostomy. Br J Ophthalmol 84(8): 903-906.

[16]. Tsai CC, Kau HC, Kao SC, Hsu WM, Liu JH (2002) Efficacy of probing the nasolacrimal duct with adjunctive Mitomycin-C for epiphora in adults. Ophthalmology 109(1): 172-174.
[17]. Tsai CC, Kao SC, Cheng CY, Kau HC, Hsu WM, et al. (2007) Oxidative stress change by systemic corticosteroid treatment among patients having active graves ophthalmopathy. Arch Ophthalmol 125(12): 1652-1656.

[18]. Tsai CC, Wu SB, Cheng CY, Kao SC, Kau HC, et al. (2010) Increased oxidative DNA damage, lipid peroxidation, and reactive oxygen species in cultured orbital fibroblasts from patients with Graves' ophthalmopathy: evidence that oxidative stress has a role in this disorder. Eye (Lond) 24(9): $1520-1525$.

[19]. Tsai CC, Wu SB, Cheng CY, Kao SC, Kau HC, et al. (2011) Increased response to oxidative stress challenge in Graves' ophthalmopathy orbital fibroblasts. Mol Vis 17: 2782-2788.

[20]. Liao SL, Huang SW (2011) Correlation of retrobulbar volume change with resected orbital fat volume and proptosis reduction after fatty decompression for Graves ophthalmopathy. Am J Ophthalmol 151(3): 465-469.

[21]. Wei YH, Chi MC, Liao SL (2011) Predictability of visual function and nerve fiber layer thickness by cross-sectional areas of extraocular muscles in graves ophthalmopathy. Am J Ophthalmol 151(5): 901-906.

[22]. Shen NY (2010) Survey on the marketing of cosmetic surgery and aesthetic surgery, Apple Daily 2010 (Taiwan); Available at http://www.appledaily. com.tw/appledaily/archive/20100810;2010 [in Chinese].

[23]. Overview of the Medicare National Physician Fee Schedule. Baltimore, MD: Centers of Medicare and Medicaid Service. Available at http://www.cms. gov/apps/physician-fee-schedule/. 